20. Schipper PH, Diggs BS, Ungerleider RM, et al. The influence of surgeon specialty on outcomes in general thoracic surgery: a national sample 1996 to 2005. Ann Thorac Surg 2009;88:1566-72;discussion 1572-63.

21. Benzo R, Kelley GA, Recchi L, et al. Complications of lung resection and exercise capacity: a meta-analysis. Respir Med 2007;101:1790-7.

22. Falcoz PE, Conti M, Brouchet L, et al. The Thoracic Surgery Scoring System (Thoracoscore): risk model for in-hospital death in 15,183 patients requiring thoracic surgery. J Thorac Cardiovasc Surg 2007; 133:325-32

23. Chamogeorgakis TP, Connery CP, Bhora F, et al. Thoracoscore predicts midterm mortality in patients undergoing thoracic surgery. J Thorac Cardiovasc Surg 2007;134:883-7.

24. Montori VM, Permanyer-Miralda G, FerreiraGonzalez I, et al. Validity of composite end points in clinical trials. Br Med J 2005;330:594-6.
25. Lim E, Brown A, Helmy A, et al. Composite outcomes in cardiovascular research: a survey of randomized trials. Ann Intern Med 2008;149:612-17.

26. Brunelli A, Socci L, Refai M, et al. Quality of life before and after major lung resection for lung cancer: a prospective follow-up analysis. Ann Thorac Surg 2007:84:410-16.

\title{
Pseudomonas aeruginosa infection in cystic fibrosis: prevent, eradicate or both?
}

\author{
Ernst Eber, Maximilian S Zach
}

Lung disease in cystic fibrosis (CF) starts early in life. Infection, inflammation, reduced lung function and abnormal chest CT findings are present in a significant proportion of infants with CF at a time when many of these children have no clinically apparent lung disease. ${ }^{1-6}$ Infection and inflammation are intimately linked early in the course of CF lung disease. ${ }^{2} 7-9$ The detection of airway inflammation in the absence of apparent infection led to the speculation that CF is associated with an intrinsic abnormality of immune regulation. ${ }^{10}$ However, more recent data argue against a primary CF-associated dysregulation of local immune function where inflammation might precede infection; they rather support the central role of bacterial pathogens in initiating and sustaining the neutrophil-mediated airway inflammation characteristic of CF lung disease, with a higher inflammatory burden in children infected with Pseudomonas aeruginosa (PA) than in those infected with organisms other than PA or uninfected. ${ }^{6} 1112$ Chronic respiratory infection is one of the main characteristics of CF and significantly contributes to morbidity and mortality. ${ }^{12}{ }^{13}$ In young children Staphylococcus aureus, Haemophilus influenzae and PA are the major lower airway pathogens. ${ }^{12} 13$ Chronic infection with PA is known to be associated with a worse prog-

Klinische Abteilung für Pulmonologie und Allergologie, Univ-Klinik für Kinder- und Jugendheilkunde, Medizinische Universität Graz, Graz, Austria

Correspondence to Dr Ernst Eber, Klinische Abteilung für Pulmonologie und Allergologie, Univ-Klinik für Kinderund Jugendheilkunde, Medizinische Universität Graz, Auenbruggerplatz 34, 8036 Graz, Austria;

ernst.eber@medunigraz.at nosis in children, adolescents and adults, and patients with mucoid PA strains do worse than those with non-mucoid strains. ${ }^{14-18}$ Thus, prevention or early detection and treatment of infection, in particular with PA, may prevent or delay irreversible lung damage from inflammation and consequently may improve prognosis. In order to prevent acquisition of PA, hygienic measures to decontaminate environmental reservoirs of this organism including medical equipment have been stressed, and CF centres have adopted meticulous microbiological surveillance and effective segregation policies to limit cross-infection between patients. Furthermore, several trials have been undertaken to assess the value of vaccination as a preventive strategy for PA infection but, with the variable outcomes of these trials, vaccines against PA cannot be recommended. ${ }^{19}$ At present, early treatment of initial infection-that is, an attempt to eradicate $\mathrm{PA}$-is the agreed standard. ${ }^{20}$ Literature on antibiotic prophylaxis of PA infection has so far remained scarce. ${ }^{21} 22$

In this issue of Thorax, TramperStranders et al report on a randomised controlled trial investigating the effectiveness of cycled antibiotic prophylaxis in children with CF (see page 915). ${ }^{23}$ Children between 0 and 18 years of age received 3 -monthly 3 -week treatments with oral ciprofloxacin and inhaled colistin or placebo for 3 years. The authors apparently chose this regimen because many CF centres across Europe have used the combination of oral ciprofloxacin and inhaled colistin as eradication therapy for $\mathrm{PA}$, going back to the first randomised controlled trial of an eradication regimen almost 20 years ago. $^{24}$ Patients were followed at 3-monthly intervals in between treatment cycles and respiratory specimens collected for microbiology studies were either oropharyngeal cough swabs or sputum samples (notably, the vast majority of patients did not produce sputum). In addition, serum samples were collected every 6 months for measurement of anti-Pseudomonas antibodies. Infection with PA (the primary end point) was defined as either two positive cultures taken $>1$ week apart or one positive culture and a 'severe pulmonary exacerbation'. Nineteen out of 65 patients (29\%) reached the primary end point (in only 4 of them was PA detected in sputum); information on how many strains were mucoid or non-mucoid is not given. Fourteen patients had positive antibody titres during the study period once or more often; 10 of them were culturepositive before they were serology-positive. This study once more shows that detection of infection in children with $\mathrm{CF}$ is difficult. In general, young children are not able to expectorate sputum, and oropharyngeal cultures do not reliably predict the presence of bacterial pathogens in the lower airways of young children with CF. ${ }^{13} 25$ Thus, cultures from bronchoalveolar lavage (BAL) fluid are usually taken as the gold standard for detection of infection in the lower airways. However, owing to sampling problems, even a negative BAL fluid culture or the absence of a particular organism on culture does not guarantee that the organism is not present in the lungs of the patient. ${ }^{26}$ For this reason, additional markers of infection are desirable and the use of specific serum antibodies against PA may be helpful in defining the status of PA infection. However, the majority of patients are antibody-negative at the time of first infection so specific antibodies against PA alone are not recommended to diagnose early infection. ${ }^{27}$ With oropharyngeal cough swabs being the prevailing respiratory specimens, one might speculate that at least some patients with positive upper airway cultures might have had negative 
cultures from lower airway specimens (and vice versa in a probably smaller number) in the present study. Thus, the reported results such as incidence rates of PA infection, mean duration to acquiring PA infection and median age at PA infection have to be interpreted with some caution.

In the study by Tramper-Stranders et al, patients with PA infection did not receive further study intervention but received 'standard anti-Pseudomonas treatment'. This treatment resulted in eradication of PA in $60 \%$ of the control group and $67 \%$ of the treatment group after one or more eradication cycles. Finally, chronic infection (defined as $>50 \%$ of evaluable months PA culture-positive) was reported for 10 of 65 patients $(15 \%)$ - that is, half of the patients with initial PA infection-at the end of the study period. ${ }^{23}$ Today there is consensus that early treatment of PA infection with the aim of eradicating the organism (and thereby achieving a reduction in airway inflammation) is worthwhile, although insufficient evidence exists to determine whether antibiotic strategies for the eradication of PA decrease mortality or morbidity, improve quality of life or are associated with adverse effects compared with placebo or standard treatment. ${ }^{20} 28$ Numerous strategies for eradication of PA have been proposed, but many questions pertaining to the selection of antibiotics, dosages, modes of delivery and duration of therapy still remain unanswered. ${ }^{28-30}$ The eradication success rate with 'standard anti-Pseudomonas treatment', as reported by Tramper-Stranders et al, appears to be relatively low when compared with the success rates in recent publications which have been reported to be around $90 \%{ }^{12}$ 30-32 With one exception, the latter studies employed cultures from BAL fluid and, in the study by Gibson et al, there was a less pronounced effect of inhaled tobramycin on the eradication of PA defined on the basis of oropharyngeal cultures compared with BAL fluid cultures. ${ }^{31}$ This further corroborates the abovementioned doubts regarding the validity of oropharyngeal cough swabs in detecting initial PA infection. As an alternative explanation, the relatively low eradication rate might partially be due to the presence of PA of the mucoid phenotype which is less amenable to treatment. ${ }^{31}$ As a side note, the rate of decline in forced expiratory volume in $1 \mathrm{~s}\left(\mathrm{FEV}_{1}\right)$ expressed as percentage predicted-approximately $2.8 \%$ per year for the control group and $3.5 \%$ per year for the treatment group (no difference between the groups)appears to be quite high compared with recent publications from Europe and the
USA, especially for the age group investigated. ${ }^{33} 34$ High baseline $\mathrm{FEV}_{1}$, which has been recognised as a significant risk factor for lung function decline, may partly explain these surprising numbers; alternatively, the patients with chronic PA infection (the latter being well known as an independent risk factor for decline in $\mathrm{FEV}_{1}$ ) may have contributed excessively to this result.

A significant weakness of the present study is that mannitol was used for placebo inhalation. Mannitol, a naturally occurring sugar alcohol, has been used as an osmotic agent and has been reported to increase mucociliary clearance since the late 1990s. ${ }^{35}$ Inhaled mannitol was recently shown to improve hydration and surface properties of sputum and lung function in patients with CF and to be at least as effective as recombinant human deoxyribonuclease (rhDNase), although with marked individual variation in both tolerance and response to treatment. ${ }^{36-38}$ Aerosolised rhDNase in patients with CF was reported to result in less positive airway cultures and a reduced demand for antibiotics. ${ }^{39}$ On the basis of the above studies, it may be speculated that inhaled mannitol acts in a similar way to rhDNase and, by improving mucociliary clearance, has some impact on PA infection. Thus, although these studies used higher dosages of mannitol than the present one and compliance in the control group might have been suboptimal due to adverse events to mannitol such as bronchoconstriction and cough, it cannot be ruled out that one active treatment has been compared with another one and not with a true placebo.

With no significant and/or clinically relevant differences found between the treatment and control groups, TramperStranders et al conclude that ' 3 -monthly cycled anti- $P$ aeruginosa prophylaxis does not reduce the risk of initial and chronic infection in $P$ aeruginosa-negative children with CF of all ages'. ${ }^{23}$ As discussed by Tramper-Stranders et al, continuous prophylaxis may be more effective in the prevention of PA infection than cycled prophylaxis. Two observational retrospective studies that used such continuous prophylaxis have been published. In the CF centre in St Luc, Belgium, 95\% of paediatric patients with CF receive intermittent or continuous inhaled antibiotic therapy (mainly tobramycin or amikacin) and this centre reports a very low rate of chronic colonisation with PA. ${ }^{22}$ In a retrospective evaluation of our practice to give prophylactic nebulised gentamicin continuously to patients with CF with defined risk situ- ations for acquisition of PA, we showed that such long-term prophylaxis can effectively delay PA infection. ${ }^{21}$ The discrepancy between these findings and the results of Tramper-Stranders et al may be interpreted as showing that any attempt at preventing or delaying PA infection in CF will be more successful when using continuous prophylaxis. Clearly, there are downsides to the long-term use of antibiotics including the emergence of other and (multi)resistant microorganisms and, particularly for aminoglycosides, side effects such as cumulative nephrotoxicity and cochleotoxicity. ${ }^{40-42}$ Over the last decade important changes have occurred in the patterns of CF airway microbiology, including increased aminoglycoside resistance and the emergence of other (multi)resistant organisms. ${ }^{42}$ Tramper-Stranders et al also describe shifts in bacterial colonisation in their treatment group. ${ }^{23}$

The authors should be applauded for undertaking the first prospective study on antibiotic prophylaxis of PA infection in children with CF. With a cycled antibiotic prophylaxis, however, this study more likely involved a mixture of prevention and early treatment of intermittent PA infection than just prevention alone. In this context, the results of the large EPIC clinical trial, which are expected to be published in the next year, will be of interest. The EPIC study compares cycled antibiotic therapy (regardless of findings from respiratory cultures) and culture-based antibiotic intervention (treatment based on recovery of PA from respiratory cultures) throughout an 18 -month study period. ${ }^{43}$ With PA being the most common and significant pathogen for patients with CF, further large-scale studies on the prevention or delay of PA infection are essential before any definite judgement can be made on the clinical value of anti-Pseudomonas prophylaxis.

\section{Competing interests None.}

Provenance and peer review Commissioned; not externally peer reviewed.

Thorax 2010;65:849-851.

doi:10.1136/thx.2010.138891

\section{REFERENCES}

1. Armstrong DS, Grimwood K, Carzino R, et al. Lower respiratory infection and inflammation in infants with newly diagnosed cystic fibrosis. BMJ 1995;310:1571-2

2. Khan TZ, Wagener JS, Bost T, et al. Early pulmonary inflammation in infants with cystic fibrosis. Am J Respir Crit Care Med 1995;151:1075-82.

3. Ranganathan SC, Bush $A$, Dezateux $C$, et al; The London Collaborative Cystic Fibrosis Group. Relative ability of full and partial forced expiratory maneuvers to identify diminished airway function in infants with cystic fibrosis. Am J Respir Crit Care Med 2002;166:1350-7. 
4. Long FR, Williams RS, Castile RG. Structural airway abnormalities in infants and young children with cystic fibrosis. J Pediatr 2004;144:154-61.

5. Linnane BM, Hall GL, Nolan G, et al; on behalf of the Australian Respiratory Early Surveillance Team for Cystic Fibrosis (AREST-CF). Lung function in infants with cystic fibrosis diagnosed by newborn screening. Am J Respir Crit Care Med 2008:178:1238-44.

6. Sly PD, Brennan S, Gangell C, et al; on behalf of the Australian Respiratory Early Surveillance Team for Cystic Fibrosis (AREST-CF). Lung disease at diagnosis in infants with cystic fibrosis detected by newborn screening. $A m J$ Respir Crit Care Med 2009;180:146-52.

7. Armstrong DS, Grimwood K, Carlin JB, et al. Lower airway inflammation in infants and young children with cystic fibrosis. Am J Respir Crit Care Med 1997:156:1197-204

8. Dakin CJ, Numa AH, Wang $\mathrm{H}$, et al. Inflammation, infection, and pulmonary function in infants and young children with cystic fibrosis. Am J Respir Crit Care Med 2002;165:904-10.

9. Sagel SD, Gibson RL, Emerson J, et al; For the Inhaled Tobramycin in Young Children Study Group and the Cystic Fibrosis Foundation Therapeutics Development Network. Impact of Pseudomonas and Staphylococcus infection on inflammation and clinical status in young children with cystic fibrosis. J Pediatr 2009:154:183-8.

10. Chmiel JF, Berger M, Konstan MW. The role of inflammation in the pathophysiology of CF lung disease. Clin Rev Allergy Immunol 2002;23:5-27.

11. Armstrong DS, Hook SM, Jamsen KM, et al. Lower airway inflammation in infants with cystic fibrosis detected by newborn screening. Pediatr Pulmonol 2005:40:500-10.

12. Douglas TA, Brennan S, Gard S, et al. Acquisition and eradication of $P$. aeruginosa in young children with cystic fibrosis. Eur Respir J 2009;33:305-11.

13. Rosenfeld M, Emerson J, Accurso F, et al. Diagnostic accuracy of oropharyngeal cultures in infants and young children with cystic fibrosis. Pediatr Pulmonol 1999;28:321-8.

14. Kosorok MR, Zeng L, West SEH, et al. Acceleration of lung disease in children with cystic fibrosis after Pseudomonas aeruginosa acquisition. Pediatr Pulmonol 2001;32:277-87.

15. Nixon GM, Armstrong DS, Carzino R, et al. Clinical outcome after early Pseudomonas aeruginosa infection in cystic fibrosis. J Pediatr 2001;138:699-704.

16. Emerson J, Rosenfeld M, McNamara S, et al. Pseudomonas aeruginosa and other predictors of mortality and morbidity in young children with cystic fibrosis. Pediatr Pulmonol 2002;34:91-100.

17. Li Z, Kosorok MR, Farrell PM, et al. Longitudinal development of mucoid Pseudomonas aeruginosa infection and lung disease progression in children with cystic fibrosis. JAMA 2005;293:581-8.

18. Courtney JM, Bradley J, Mccaughan J, et al. Predictors of mortality in adults with cystic fibrosis. Pediatr Pulmonol 2007;42:525-32

19. Johansen HK, Gøtzsche PC. Vaccines for preventing infection with Pseudomonas aeruginosa in cystic fibrosis. Cochrane Database Syst Rev 2008;(4): CD001399.

20. Döring G, Høiby N; For the Consensus Study Group. Early intervention and prevention of lung disease in cystic fibrosis: a European consensus. J Cyst Fibros 2004;3:67-91

21. Heinzl B, Eber E, Oberwaldner B, et al. Effects of inhaled gentamicin prophylaxis on acquisition of Pseudomonas aeruginosa in children with cystic fibrosis: a pilot study. Pediatr Pulmonol 2002:33:32-7.

22. Lebecque $\mathbf{P}$, Leal T, Zylberberg $\mathrm{K}$, et al. Towards zero prevalence of chronic Pseudomonas aeruginosa infection in children with cystic fibrosis. J Cyst Fibros 2006;5:237-44.

23. Tramper-Stranders GA, Wolfs TFW, van Haren Noman S, et al. Controlled trial of cycled antibiotic prophylaxis to prevent initial Pseudomonas aeruginosa infection in children with cystic fibrosis. Thorax 2010;65:897-902

24. Valerius N, Koch C, Høiby N. Prevention of chronic Pseudomonas aeruginosa colonisation in cystic fibrosis by early treatment. Lancet 1991;338:725-6.

25. Armstrong DS, Grimwood K, Carlin JB, et al. Bronchoalveolar lavage or oropharyngeal cultures to identify lower respiratory pathogens in infants with cystic fibrosis. Pediatr Pulmonol 1996;21:267-75.

26. Gutierrez JP, Grimwood K, Armstrong DS, et al. Interlobar differences in bronchoalveolar lavage fluid from children with cystic fibrosis. Eur Respir $\mathrm{J}$ 2001:17:281-6

27. Ratjen $\mathbf{F}$, Walter $H$, Haug $M$, et al. Diagnostic value of serum antibodies in early Pseudomonas aeruginosa infection in cystic fibrosis patients. Pediatr Pulmonol 2007:42:249-55.

28. Langton Hewer SC, Smyth AR. Antibiotic strategies for eradicating Pseudomonas aeruginosa in people with cystic fibrosis. Cochrane Database Syst Rev 2009; (4):CD004197.

29. Jones AM. Eradication therapy for early Pseudomonas aeruginosa infection in CF: many questions still unanswered. Eur Respir $\mathrm{J}$ 2005;26:373-5

30. Eber $\mathbf{E}$, Thalhammer GH, Zach MS. Eradication of Pseudomonas aeruginosa in cystic fibrosis. Eur Respir J 2006;27:438-9

31. Gibson RL, Emerson J, Mayer-Hamblett N, et al; For the Inhaled Tobramycin in Young Children Study
Group and the Cystic Fibrosis Foundation Therapeutics Development Network. Duration of treatment effect after tobramycin solution for inhalation in young children with cystic fibrosis Pediatr Pulmonol 2007;42:610-23.

32. Ratjen F, Munck $A$, Kho $P$, et al; For the ELITE Study Group. Treatment of early Pseudomonas aeruginosa infection in patients with cystic fibrosis: the ELITE trial. Thorax 2010;65:286-91.

33. Que C, Cullinan P, Geddes D. Improving rate of decline of FEV1 in young adults with cystic fibrosis. Thorax 2006;61:155-7.

34. Konstan MW, Morgan WJ, Butler SM, et al; For the Scientific Advisory Group and the Investigators and Coordinators of the Epidemiologic Study of Cystic Fibrosis. Risk factors for rate of decline in forced expiratory volume in one second in children and adolescents with cystic fibrosis. J Pediatr 2007;151:134-9.

35. Daviskas E, Anderson SD, Brannan JD, et al. Inhalation of dry-powder mannitol increases mucociliary clearance. Eur Respir J 1997; 10:2449-54.

36. Jaques A, Daviskas E, Turton JA, et al. Inhaled mannitol improves lung function in cystic fibrosis. Chest 2008:133:1388-96.

37. Daviskas E, Anderson SD, Jaques A, et al. Inhaled mannitol improves the hydration and surface properties of sputum in patients with cystic fibrosis. Chest 2010;137:861-8.

38. Minasian C, Wallis C, Metcalfe $\mathrm{C}$, et al. Comparison of inhaled mannitol, daily rhDNase and a combination of both in children with cystic fibrosis: a randomised trial. Thorax 2010;65:51-6

39. Frederikson B, Pressler T, Hansen A, et al. Effect of aerosolized rhDNase (Pulmozyme ${ }^{\circledR}$ ) on pulmonary colonization in patients with cystic fibrosis. Acta Pædiatr 2006;95:1070-4.

40. Ring $\mathbf{E}$, Eber $\mathrm{E}$, Erwa $\mathrm{W}$, et al. Urinary N-acetyl- $\beta$-Dglucosaminidase activity in patients with cystic fibrosis on long term gentamicin inhalation. Arch Dis Child 1998;78:540-3.

41. Bockenhauer D, Hug MJ, Kleta R. Cystic fibrosis, aminoglycoside treatment and acute renal failure: the not so gentle micin. Pediatr Nephrol 2009;24:925-8.

42. Emerson J, McNamara S, Buccat AM, et al. Changes in cystic fibrosis sputum microbiology in the United States between 1995 and 2008. Pediatr Pulmonol 2010:45:363-70.

43. Treggiari MM, Rosenfeld M, Mayer-Hamblett N, et al; EPIC Study Group. Early anti-pseudomonal acquisition in young patients with cystic fibrosis: rationale and design of the EPIC clinical trial and observational study. Contemp Clin Trials 2009;30:256-68.

\section{Relearning an old lesson: stopping trials early}

\section{Najib M Rahman, Robert J 0 Davies}

\author{
UKCRC Oxford Respiratory Trials Unit, University of \\ Oxford and Oxford Centre for Respiratory Medicine, \\ Oxford Radcliffe Hospital, Oxford, UK \\ Correspondence to Robert J 0 Davies, Oxford Radcliffe \\ Hospital and Oxford University, Churchill Hospital, \\ Headington, 0xford OX3 7LJ, UK; \\ robert.davies@ndm.ox.ac.uk
}

A well designed and delivered clinical trial is the main tool to define whether medical interventions 'work', and how well. As such, they are potent weapons in the armoury of medical progress - and like all potent weapons need to be used with care.
In this month's Thorax, Koegelenberg et al (see page 857) report the findings of a trial comparing the diagnostic accuracy of closed pleural biopsy (Abrams needle) and cutting needle pleural biopsy after thoracic ultrasound, for the diagnosis of pleural tuberculosis (TB). ${ }^{1}$ This question is clearly important given the global significance of $\mathrm{TB}$, and the key role of pleural biopsy in the diagnosis and microbiological assessment of its pleural presentations. To date, there are no published studies assessing ultrasoundguided pleural biopsy for the diagnosis of 